UDC 517.537 .72

\author{
M. M. SHeREMETA
}

\title{
NOTE TO THE BEHAVIOR OF THE MAXIMAL TERM OF DIRICHLET SERIES ABSOLUTELY CONVERGENT IN HALF-PLANE
}

M. M. Sheremeta. Note to the behavior of the maximal term of Dirichlet series absolutely convergent in half-plane, Mat. Stud. 56 (2021), 144-148.

Denote by $S_{0}(\Lambda)$ the class of Dirichlet series $F(s)=\sum_{n=0}^{\infty} a_{n} \exp \left\{s \lambda_{n}\right\}(s=\sigma+i t)$ with an increasing to $+\infty$ sequence $\Lambda=\left(\lambda_{n}\right)$ of exponents $\left(\lambda_{0}=0\right)$ and the abscissa of absolute convergence $\sigma_{a}=0$. We say that $F \in S_{0}^{*}(\Lambda)$ if $F \in S_{0}(\Lambda)$ and $\ln \lambda_{n}=o\left(\ln \left|a_{n}\right|\right)(n \rightarrow \infty)$. Let $\mu(\sigma, F)=\max \left\{\left|a_{n}\right| \exp \left(\sigma \lambda_{n}\right): n \geq 0\right\}$ be the maximal term of Dirichlet series. It is proved that in order that

$$
\ln (1 /|\sigma|)=o(\ln \mu(\sigma))(\sigma \uparrow 0)
$$

for every function $F \in S_{0}^{*}(\Lambda)$ it is necessary and sufficient that

$$
\varlimsup_{n \rightarrow \infty} \frac{\ln \lambda_{n+1}}{\ln \lambda_{n}}<+\infty .
$$

For an analytic in the disk $\{z:|z|<1\}$ function $f(z)=\sum_{n=0}^{\infty} a_{n} z^{n}$ and $r \in(0,1)$ we put $M_{f}(r)=\max \{|f(z)|:|z|=r<1\}$ and $\mu_{f}(r)=\max \left\{\left|a_{n}\right| r^{n}: n \geq 0\right\}$. As a corollary we get the following statement: if there exists a sequence $\left(n_{j}\right)$ such that

$$
\ln n_{j+1}=O\left(\ln n_{j}\right) \text { and } \ln n_{j}=o\left(\ln \left|a_{n_{j}}\right|\right) \text { as } j \rightarrow \infty,
$$

then the functions $\ln \mu_{f}(r)$ and $\ln M_{f}(r)$ are or are not slowly increasing simultaneously.

1. Introduction. For an analytic in the disk $\{z:|z|<1\}$ function

$$
f(z)=\sum_{n=0}^{\infty} a_{n} z^{n}, \quad z=r e^{i \theta}
$$

let $M_{f}(r)=\max \{|f(z)|:|z|=r<1\}$ and $\mu_{f}(r)=\max \left\{\left|a_{n}\right| r^{n}: n \geq 0\right\}$ be the maximal term. A positive continuous and increasing to $+\infty$ on $[0,1)$ function $l$ is called slowly increasing if $l((x+1) / 2)) \sim l(x)$ as $x \uparrow 1$. It is known [1] that if

$$
\ln \frac{1}{1-r}=o\left(\ln \mu_{f}(r)\right), \quad r \uparrow 1,
$$

then $\ln \mu_{f}(r)$ and $\ln M_{f}(r)$ are or are not slowly increasing simultaneously. If the condition (2) does not hold then [1] the slow growth of $\ln M_{f}(r)$ does not follow from the slow growth of $\ln \mu_{f}(r)$, and vice versa [2]. The following question arises: under which conditions on $a_{n}$ the relation (2) is true?

2010 Mathematics Subject Classification: 30B50.

Keywords: Dirichlet series; maximal term.

doi:10.30970/ms.56.2.144-148

(C) M. M. Sheremeta, 2021 
If $\ln \left|a_{n}\right| \leq K \ln n\left(n \geq n_{0}\right)$ then

$$
\ln \mu_{f}(r) \leq \max \{K \ln t+t \ln r: t \geq 1\}+O(1)=K \ln \frac{1}{-\ln r}+O(1)=K \ln \frac{1}{1-r}+O(1)
$$

as $r \uparrow 1$. Therefore, in order that (2) holds, it is necessary that $\ln n_{k}=o\left(\ln \left|a_{n_{k}}\right|\right)$ $(k \rightarrow+\infty)$ for some increasing sequence $\left(n_{k}\right)$ of integers, and our question is reduced to finding of conditions on this sequence $\left(n_{k}\right)$. A result proved below for Dirichlet series absolutely convergent in half-plane implies that such condition is $\ln n_{k+1}=O\left(\ln n_{k}\right)(k \rightarrow \infty)$.

2. Main result. So, let $\Lambda=\left(\lambda_{n}\right)$ be an increasing to $+\infty$ sequence of positive numbers $\left(\lambda_{0}=0\right)$, and Dirichlet series

$$
F(s)=\sum_{n=0}^{\infty} a_{n} \exp \left\{s \lambda_{n}\right\}, \quad s=\sigma+i t,
$$

has the abscissa of absolute convergence $\sigma_{a}=0$. For $\sigma<0$ let

$$
\mu(\sigma, F)=\max \left\{\left|a_{n}\right| \exp \left(\sigma \lambda_{n}\right): n \geq 0\right\}
$$

be the maximal term of series (3). We investigate conditions on $\left(a_{n}\right)$ and $\left(\lambda_{n}\right)$, under which

$$
\ln \frac{1}{|\sigma|}=o(\ln \mu(\sigma)), \quad \sigma \uparrow 0 .
$$

To that end we denote by $S_{0}^{*}(\Lambda)$ the class of Dirichlet series (3) absolutely convergent in the half-plane $\{s: \operatorname{Re} \sigma<0\}$ such that $\ln \lambda_{n}=o\left(\ln \left|a_{n}\right|\right)(n \rightarrow \infty)$.

Theorem 1. In order that (4) holds for every function $F \in S_{0}^{*}(\Lambda)$, it is necessary and sufficient that

$$
\varlimsup_{n \rightarrow \infty} \frac{\ln \lambda_{n+1}}{\ln \lambda_{n}}<+\infty .
$$

Proof. Let us start with the sufficiency. Let $\Omega(0)$ be the class of positive unbounded on $(-\infty, 0)$ functions $\Phi$ such that the derivative $\Phi^{\prime}$ is positive, continuously differentiable and increasing to $+\infty$ on $(-\infty, 0)$. We denote by $\varphi$ the inverse function to $\Phi^{\prime}$, and let $\Psi(x)=x-\Phi(x) / \Phi^{\prime}(x)$ be the function associated with $\Phi$ in the sense of Newton. It is clear that the function $\varphi$ is continuously differentiable and increasing to 0 on $(0,+\infty)$. The function $\Psi$ is $([3,4],[5$, p.30]) continuously differentiable and increasing to 0 on $(-\infty, 0)$.

For $\Phi \in \Omega(0)$ and $0 \leq a<b<+\infty$ we put

$$
G_{1}(a, b, \Phi)=\frac{a b}{b-a} \int_{a}^{b} \frac{\Phi(\varphi(t))}{t^{2}} d t, \quad G_{2}(a, b, \Phi)=\Phi\left(\frac{1}{b-a} \int_{a}^{b} \varphi(t) d t\right) .
$$

Then ([6], [5, p.34]) $G_{1}(a, b, \Phi)<G_{2}(a, b, \Phi)$. It is clear that $G_{2}\left(\lambda_{n}, \lambda_{n+1}, \Phi\right)=\Phi\left(\varkappa_{n}\right)$, where

$$
\varkappa_{n}=\frac{1}{\lambda_{n+1}-\lambda_{n}} \int_{\lambda_{n}}^{\lambda_{n+1}} \varphi(t) d t .
$$

Theorem 3.1 in [4], [5, p. 34-35] implies that if $\ln \left|a_{n}\right| \geq-\lambda_{n} \Psi\left(\varphi\left(\lambda_{n}\right)\right)\left(n \geq n_{0}\right)$ then

$$
\ln \mu(\sigma, F) \geq \Phi(\sigma) \frac{G_{1}\left(\lambda_{n}, \lambda_{n+1}, \Phi\right)}{G_{2}\left(\lambda_{n}, \lambda_{n+1}, \Phi\right)}
$$


for all $\sigma \in\left[\varphi\left(\lambda_{n}\right), \varphi\left(\lambda_{n+1}\right)\right]$ and $n \geq n_{0}$. We remark also that if a function $f$ is positive, continuous and increasing to $+\infty$ on $[0,+\infty)$ such that $f(x)>x$ and $\lambda_{n+1} \leq f\left(\lambda_{n}\right)$ then $([7],[5$, p. 34])

$$
\frac{G_{1}\left(\lambda_{n}, \lambda_{n+1}, \Phi\right)}{G_{2}\left(\lambda_{n}, \lambda_{n+1}, \Phi\right)} \geq \frac{G_{1}\left(\lambda_{n}, f\left(\lambda_{n}\right), \Phi\right)}{G_{2}\left(\lambda_{n}, f\left(\lambda_{n}\right), \Phi\right)} .
$$

Now, let $T>0$ be an arbitrary number and $\Phi(\sigma)=T \ln \frac{1}{|\sigma|}$. Then $\varphi(x)=-\frac{T}{x}, \Psi(\sigma)=$ $-|\sigma| \ln \frac{e}{|\sigma|}$, and $\Psi(\varphi(x))=-\frac{T}{x} \ln \frac{e x}{T}$. Therefore,

$$
G_{1}\left(\lambda_{n}, \lambda_{n+1}, \Phi\right)=T \frac{\lambda_{n+1} \ln \lambda_{n}-\lambda_{n} \ln \lambda_{n+1}}{\lambda_{n+1}-\lambda_{n}}+T \ln \frac{e}{T}
$$

and

$$
G_{2}\left(\lambda_{n}, \lambda_{n+1}, \Phi\right)=T \ln \frac{\lambda_{n+1}-\lambda_{n}}{\ln \lambda_{n+1}-\ln \lambda_{n}}-T \ln T
$$

From the definition of $S^{*}(\Lambda)$ we have $\ln \left|a_{n}\right| \geq T \ln \lambda_{n} \geq T \ln \left(e \lambda_{n} / T\right)=-\lambda_{n} \Psi\left(\varphi\left(\lambda_{n}\right)\right)$ for arbitrary $T \geq e$ and all $n \geq n_{0}(T)$, and from condition (5) it follows that there exists a number $\beta>0$ such that $\lambda_{n+1} \leq \lambda_{n}^{1+\beta}\left(n \geq n_{0}\right)$. Therefore, (7) implies

$$
\frac{G_{1}\left(\lambda_{n}, \lambda_{n+1}, \Phi\right)}{G_{2}\left(\lambda_{n}, \lambda_{n+1}, \Phi\right)} \geq \frac{G_{1}\left(\lambda_{n}, \lambda_{n}^{1+\beta}, \Phi\right)}{G_{2}\left(\lambda_{n}, \lambda_{n}^{1+\beta}, \Phi\right)}=\frac{\frac{\lambda_{n}^{1+\beta} \ln \lambda_{n}-(1+\beta) \lambda_{n} \ln \lambda_{n}}{\lambda_{n}^{1+\beta}-\lambda_{n}}-\ln \frac{T}{e}}{\ln \frac{\lambda_{n}^{1+\beta}-\lambda_{n}}{\beta \ln \lambda_{n}}-\ln T}=\frac{1+o(1)}{1+\beta}
$$

as $n \rightarrow \infty$ and, thus, from (6) we get

$$
\ln \mu(\sigma, F) \geq \frac{(1+o(1)) T}{1+\beta} \ln \frac{1}{|\sigma|}, \quad \sigma \uparrow 0 .
$$

i. e. in view of the arbitrariness of $T$ we obtain (4).

Now we prove the necessity. Suppose that condition (5) does not hold, i. e. there exists an increasing to $+\infty$ sequence of integers such that $\ln \lambda_{n_{k}+1} / \ln \lambda_{n_{k}} \rightarrow \infty, k \rightarrow \infty$. We choose a slowly increasing to $+\infty$ on $[0,+\infty)$ continuously differentiable function $\alpha$ such that $\alpha\left(\ln \lambda_{n_{k+1}}\right) \leq \frac{\ln \lambda_{n_{k}+1}}{\ln \lambda_{n_{k}}}\left(k \geq k_{0}\right)$ and the function $\Phi(\sigma)=\alpha\left(\ln \frac{1}{|\sigma|}\right) \ln \frac{1}{|\sigma|}$ belongs to $\Omega(0)$. We choose the coefficients of Dirichlet series such that $\ln \left|a_{n}\right|=-\lambda_{n} \Psi\left(\varphi\left(\lambda_{n}\right)\right)$. Then $\varkappa_{n}=\frac{\ln \left|a_{n}\right|-\ln \left|a_{n+1}\right|}{\lambda_{n+1}-\lambda_{n}}$, because $(x \Psi(\varphi(x)))^{\prime}=\varphi(x)$. Since the function $\alpha$ is slowly increasing, we have $x \alpha^{\prime}(x) / \alpha(x) \rightarrow 0$ as $x \rightarrow+\infty$. Therefore,

$$
\Phi^{\prime}(\sigma)=\frac{1}{|\sigma|}\left\{\alpha^{\prime}\left(\ln \frac{1}{|\sigma|}\right) \ln \frac{1}{|\sigma|}+\alpha\left(\ln \frac{1}{|\sigma|}\right)\right\}=\frac{1+o(1)}{|\sigma|} \alpha\left(\ln \frac{1}{|\sigma|}\right), \quad \sigma \uparrow 0,
$$

and in order to find the asymptotical behaviour of $\varphi$ it is necessary to solve the equation

$$
\ln \frac{1}{|\sigma|}+\ln \alpha\left(\ln \frac{1}{|\sigma|}\right)=\ln x+o(1), \quad x \rightarrow+\infty
$$

We find a solution $\sigma=\sigma(x)$ of (8) in the form

$$
\ln \frac{1}{|\sigma|}=\ln x-\beta, \quad \beta=\beta(x)=o(\ln x), \quad x \rightarrow+\infty .
$$


Substituting (9) in (8) we obtain $\beta=\ln \alpha(\ln x-\beta)+o(1), x \rightarrow+\infty$. But for some $\xi \in$ $(\ln x-\beta, \ln x)$ we have $\alpha(\ln x)-\alpha(\ln x-\beta)=\alpha^{\prime}(\xi) \beta=o\left(\xi \alpha^{\prime}(\xi)\right)=o(\alpha(\xi))=o(\alpha(\ln x))$ $(x \rightarrow+\infty)$, i.e. $\beta(x)=\ln \alpha(\ln x)+o(1), x \rightarrow+\infty$, and, therefore, from (9) we obtain $\ln \frac{1}{|\sigma|}=\ln x-\ln \alpha(\ln x)+o(1), x \rightarrow+\infty$. Thus,

$$
\varphi(x)=-\frac{(1+o(1)) \alpha(\ln x)}{x}, \quad x \rightarrow+\infty .
$$

Using L'Hôspitale rule and relation (10) we see that Dirichlet series (3) with choosen coefficients belongs to $S_{0}^{*}(\Lambda)$. From (10) it follows also that

$$
\begin{gathered}
\varkappa_{n_{k}}=\frac{1}{\lambda_{n_{k}+1}-\lambda_{n_{k}}} \int_{\lambda_{n_{k}}}^{\lambda_{n_{k}+1}} \varphi(x) d x=-\frac{1+o(1)}{\lambda_{n_{k}+1}-\lambda_{n_{k}}} \int_{\lambda_{n_{k}}}^{\lambda_{n_{k}+1}} \frac{\alpha(\ln x)}{x} d x \geq \\
\geq-\frac{(1+o(1)) \alpha\left(\ln \lambda_{n_{k}+1}\right)\left(\ln \lambda_{n_{k}+1}-\ln \lambda_{n_{k}}\right)}{\lambda_{n_{k}+1}-\lambda_{n_{k}}}=-\frac{(1+o(1)) \alpha\left(\ln \lambda_{n_{k}+1}\right) \ln \lambda_{n_{k}+1}}{\lambda_{n_{k}+1}}, \\
\ln \frac{1}{\left|\varkappa_{n_{k}}\right|} \geq \ln \frac{\lambda_{n_{k}+1}}{\alpha\left(\ln \lambda_{n_{k}+1}\right) \ln \lambda_{n_{k}+1}}+o(1)=(1+o(1)) \ln \lambda_{n_{k}+1}
\end{gathered}
$$

as $k \rightarrow+\infty$. On the other hand, since [4]

$$
\ln \mu\left(\varkappa_{n}, F\right)=-\lambda_{n} \Psi\left(\varphi\left(\lambda_{n}\right)\right)+\varkappa_{n} \lambda_{n}=G_{1}\left(\lambda_{n}, \lambda_{n+1}, \Phi\right)
$$

and in view of $(10)$

$$
\Phi(\varphi(x))=\alpha(\ln x-\ln \alpha(\ln x)+o(1))(\ln x-\ln \alpha(\ln x)+o(1))=(1+o(1)) \alpha(\ln x) \ln x
$$

as $x \rightarrow+\infty$, we get

$$
\begin{gathered}
\ln \mu\left(\varkappa_{n_{k}}, F\right)=(1+o(1)) \lambda_{n_{k}} \int_{\lambda_{n_{k}}}^{\lambda_{n_{k}+1}} \frac{\Phi(\varphi(x))}{x^{2}} d x= \\
=(1+o(1)) \lambda_{n_{k}} \int_{\lambda_{n_{k}}}^{\lambda_{n_{k}+1}} \frac{\alpha(\ln x) \ln x}{x^{2}} d x \leq(1+o(1)) \lambda_{n_{k}} \alpha\left(\ln \lambda_{n_{k}+1}\right) \int_{\lambda_{n_{k}}}^{\lambda_{n_{k}+1}} \frac{\ln x}{x^{2}} d x= \\
=(1+o(1)) \lambda_{n_{k}} \alpha\left(\ln \lambda_{n_{k}+1}\right)\left(\frac{\ln \lambda_{n_{k}}+1}{\lambda_{n_{k}}}-\frac{\ln \lambda_{n_{k}+1}+1}{\lambda_{n_{k}+1}}\right)= \\
=(1+o(1)) \alpha\left(\ln \lambda_{n_{k+1}}\right) \ln \lambda_{n_{k}}, \quad k \rightarrow \infty .
\end{gathered}
$$

From (11) and (12) it follows that

$$
\frac{\ln \mu\left(\varkappa_{n_{k}}, F\right)}{\ln \left(1 /\left|\varkappa_{n_{k}}\right|\right.} \leq(1+o(1)) \frac{\alpha\left(\ln \lambda_{n_{k+1}}\right) \ln \lambda_{n_{k}}}{\ln \lambda_{n_{k}+1}} \leq 1+o(1), \quad k \rightarrow \infty,
$$

i. e. relation (4) does not hold. The necessity of condition (5) is proved.

3. Corollaries. Since $\max \left\{\left|a_{n}\right| \exp \left(\sigma \lambda_{n}\right): n \geq 0\right\} \geq \max \left\{\left|a_{n_{j}}\right| \exp \left(\sigma \lambda_{n_{j}}\right): j \geq 1\right\}$ for any sequence $\left(n_{j}\right)$, Theorem 1 implies the following statement. 
Corollary 1. If there exists a subsequence $\left(\lambda_{n_{j}}\right)$ of the sequence $\left(\lambda_{n}\right)$ such that $\ln \lambda_{n_{j+1}}=O\left(\ln \lambda_{n_{j}}\right)$ and $\ln \lambda_{n_{j}}=o\left(\ln \left|a_{n_{j}}\right|\right)$ as $j \rightarrow \infty$ then (4) holds.

If in power series (1) we make the substitution $z=e^{s}$ then we obtain Dirichlet series (3) with $\lambda_{n}=n,|\sigma|=|\ln r|=(1+o(1))(1-r), r \uparrow 1$, and $\mu_{f}(r, F)=\mu(\ln r, F)$. Therefore, if there exists a sequence $\left(n_{j}\right)$ such that $\ln n_{j+1}=O\left(\ln n_{j}\right)$ and $\ln n_{j}=o\left(\ln \left|a_{n_{j}}\right|\right)$ as $j \rightarrow \infty$ then (2) holds. Hence and from above-mentioned result in [1] the following corollary follows.

Corollary 2. If there exists a sequence $\left(n_{j}\right)$ such that

$$
\ln n_{j+1}=O\left(\ln n_{j}\right) \text { and } \ln n_{j}=o\left(\ln \left|a_{n_{j}}\right|\right) \text { as } j \rightarrow+\infty,
$$

then the functions $\ln \mu_{f}(r)$ and $\ln M_{f}(r)$ are or are not slowly increasing simultaneously. In particular, if $\ln n=o\left(\ln \left|a_{n}\right|\right)$ as $n \rightarrow \infty$ then the functions $\ln \mu_{f}(r)$ and $\ln M_{f}(r)$ are or are not slowly increasing simultaneously.

\section{REFERENCES}

1. M.M. Sheremeta, M.V. Zabolotskyi, Slow growth of power series convergent in the unit disk, Mat. Stud., 11 (1999), №2, 221-224.

2. P.V. Filevych, On the slow growth of power series convergent in the unit disk, Mat. Stud., 16 (2001), №2, $217-221$.

3. M.M. Sheremeta, S.I. Fedynyak, On the derivative of Dirichlet series, Sibirsk. mat. journ., 39 (1998), №1, 206-223. (in Russian)

4. M.M. Sheremeta, O.M. Sumyk, Connection between the growth of conjugated by Young functions, Mat. Stud., 11 (1999), №2, 221-224. (in Ukrainian)

5. M.M. Sheremeta, Asymptotic behavior of Laplace-Stieltjes integrals, VNTL Publishers, 2010.

6. M.V. Zabolotskyi, M.M. Sheremeta, Generalization of Lindelöf's theorem, Ukr. Math. Zh., 50 (1998), №1, 1177-1197. (in Ukrainian)

7. O.M. Sumyk, Estimates from below of the maximal term of Dirichlet series, Visnyk Lviv Univer. Series Mech. Math., 53 (1999), 40-44. (in Ukrainian)

Ivan Franko National University of Lviv

Lviv, Ukraine

m.m.sheremeta@gmail.com

Received 19.05.2021

Revised 17.10.2021 